Proceedings

\title{
Evaluation of Low Cost Piezoelectric Sensors for the Identification of Partial Discharges Evolution ${ }^{\dagger}$
}

\author{
Daniele F. Akiyoshi ${ }^{1}$, Bruno A. de Castro ${ }^{1, *}$, José V. F. Leão ${ }^{1}$, Marco A. Rocha ${ }^{1}$, \\ Jorge A. A. Rey ${ }^{2}$, Rudolf R. Riehl ${ }^{1}$ and André L. Andreoli ${ }^{1}$ \\ 1 Department of Electrical Engineering, São Paulo State University (UNESP), 17033-360 Bauru-SP, Brazil; \\ daniele.akiyoshi@unesp.br (D.F.A.); vital@unilins.edu.br (J.V.F.L.); marco.rocha@unesp.br (M.A.R.); \\ rudolf.riehl@unesp.br (R.R.R.); andre.andreoli@unesp.br (A.L.A.) \\ 2 Department of Electrical Engineering, Universidad Técnica Federico Santa María, Av. Vicuña Mackenna \\ 3939, Santiago de Chile 8940000, Chile; jorge.ardila@usm.cl \\ * Correspondence: bruno.castro@unesp.br; Tel.: +55-14-3103-6115 \\ + Presented at the 5th International Electronic Conference on Sensors and Applications, 15-30 November \\ 2018; Available online: https://ecsa-5.sciforum.net.
}

Published: 14 November 2018

\begin{abstract}
Transformers are essential equipment in electrical energy systems and their failure may lead to the loss of a power supply. Both industry and science have sought to develop sensors and low-cost solutions for the correct diagnosis of their failures. Thus, the use of piezoelectric sensors in the diagnosis of partial discharge in power transformers has been growing significantly, in order to ensure the reduction of maintenance costs, as well as the quality of electric power supply, since this type of failure can lead to a significant cost of repair. In many cases, when partial discharge is detected, there is no immediate need to promote transformer maintenance. In this way, it becomes reasonable to study the evolution of this phenomenon, so that the maintenance of the device can be scheduled and performed correctly. In this regard, this article presents a feasibility study of a lowcost piezoelectric transducer for the identification of the evolution level of partial discharges. For this purpose, in a $30 \mathrm{kVA}$ distribution transformer, three corona partial discharges were produced under three different voltage levels, using a copper electrode. The low cost piezoelectric sensor was coupled to the transformer housing. The acoustic emission signals of the three partial discharge levels were captured and analyzed by the use of acoustic signal metrics, such as energy, peak value, and power spectral density. The experimental results indicated that the low cost sensor is able to identify the evolution of the partial discharge intensity, since the values obtained by the metrics are directly related to the partial discharge levels. Therefore, the results reported in this study indicate that the piezoelectric transducer has a great applicability in diagnosing the partial discharges evolution, and, thus, can assist in the planning of electrical maintenance.
\end{abstract}

Keywords: piezoelectric sensors; low-cost sensor; partial discharge; evolution; signal processing analysis

\section{Introduction}

Power transformers are essential equipment in electrical energy systems and their failure can affect the quality of electric power supply or may lead to lack of it, resulting in losses to consumers and electric companies [1-4]. Therefore, nowadays, both industry and science have sought to develop sensors and low-cost solutions for the correct diagnosis of their operational condition. One of the most frequent failures in transformers is partial discharge (PD), which might be the result of damage in their insulation system. It is characterized by the emission of light, heat, electromagnetic, and acoustic waves. There are a variety of PD detection methods in the specialized literature and they are 
classified as electrical, optical, chemical, and acoustical detection [1-4]. A well-known method is the acoustic emission, which employs sensors to capture the acoustic signals generated by PD [5,6].

In many cases, when a PD is detected, there is no immediate need to promote transformer maintenance. However, it is necessary to know when this failure becomes critical, so the maintenance of the device can be scheduled and performed correctly. In this way, many failures in transformers can be avoided with implementation of monitoring systems, that enable the failure evolution and then plan futures actions, such as preventive maintenance or replacement of existing equipment. Nevertheless, the sensors typically used for this purpose still have high financial costs, which makes them unfeasible for constant monitoring during the life time of a transformer [2-4]. Therefore, engineering have shown interest in developing low cost solutions, in order to make the monitoring systems more accessible, and to provide correct and precise diagnoses for failure in this type of equipment.

For this purpose, the piezoelectric sensors have become an economic alternative and presented similar results with regard to the transducers commonly used [3,4]. Thus, the use of these sensors has been growing significantly in this field, aiming to monitor the PD evolution.

Accordingly, the present study proposes a feasibility evaluation to identify the evolution level of a PD, using a piezoelectric sensor to detect the acoustic signals emitted.

The outline of this article is as follows: Sections 1.1,1.2, and 1.3, respectively, present the basic concepts of partial discharges, piezoelectric transducers, and digital signal processing analysis. The experimental setup is described in Section 2. In Section 3, the results are discussed, and the conclusion of this paper is presented in Section 4 .

\subsection{Partial Discharge in Power Transformers}

As mentioned, one of the most common faults in power transformers is partial discharge (PD). According to IEC-60270 (2011), a PD is defined as an electric discharge that partially fills the insulation between two conductors. They can arise when electric charges are created, due to the difference of electric potential in bubbles of gases, broken or degraded insulating materials, resulting in an incomplete ionized path with the appearance of free electrons [7-9]. Partial discharges enforce a disturbance in the material, characterized by the emission of heat, light, electromagnetic radiation, and ultrasonic waves in the form of pulses that propagate in all directions of the discharge source [911]. Thus, a PD can be detected by measuring and analyzing its signals and changes. The detection and the evolution identification of PD is greatly relevant, since the occurrence of these failures can be a primordial indicator of problems in the insulation system, and the progression of this phenomenon can cause a total failure of the transformers [12].

\subsection{Piezoelectric Sensors}

The piezoelectric effect occurs in materials that, when subjected to a mechanical stress, produce an output voltage (direct effect). The reverse effect also occurs, i.e., by applying an electric voltage in the piezoelectric material, a mechanical deformation arises. The equations, and more details can be accessed in $[13,14]$. The piezoelectric transducers, shown in Figure 1, have a ceramic disk of $25.0 \mathrm{~mm}$ $\times 0.23 \mathrm{~mm}$, and a brass plate of $35.0 \mathrm{~mm} \times 0.30 \mathrm{~mm}$ are already consolidated for partial discharge detection in power transformers [13]. However, this sensor was not validated for the diagnosis of the evolution of the partial discharge phenomenon. 

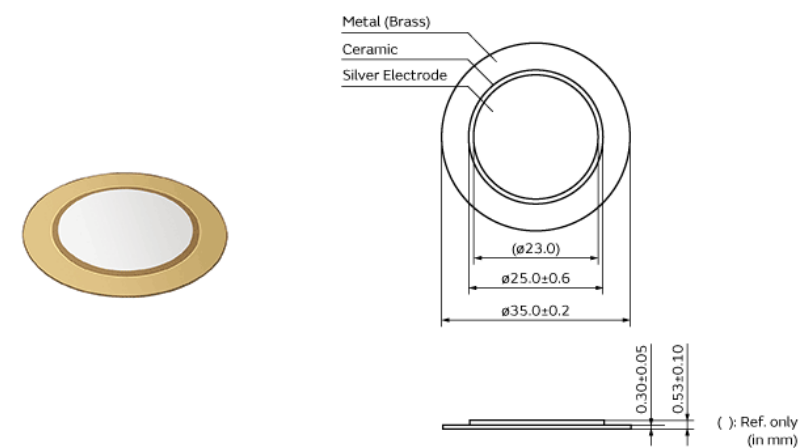

Figure 1. Murata piezoelectric sensor 7BB-35-3 used in the experiment.

In this way, some signal processing techniques, in the time and frequency domain, were applied to verify the feasibility of the piezoelectric sensors application in the evolution of this failure. The following section presents the metrics used.

\subsection{Acoustic Signal Analysis (Energy and Spectral Density)}

The parameters used to process the signals obtained in the tests were: Energy content $E_{b}$ and power spectral density $\hat{S}(\omega)^{i}$. The energy content of the signal $f(t)$ can be expressed by Equation (1), which $f(w)$ is a FFT of the $f(t)$ [15]:

$$
E_{b}=\int|f(t)|^{2} d t=\frac{1}{2 \pi} \int|f(\omega)|^{2} d \omega
$$

The power spectral density criterion (PSD) is a standard method to extract the characteristics of stochastic signals and describe the energy distribution of a signal in the frequency domain [2]. In this method, a signal $x(n)$ is divided into $L$ equal segments of length $M$. The PSD of a segment, $i$ is given by Equation (2) [3]:

$$
\hat{S}(\omega)^{i}=\frac{1}{\sum_{n=0}^{M-1} d_{M}^{2}(n)}\left|\sum_{n=0}^{M-1} x^{i}(n) d_{M} e^{-j \omega n}\right|^{2}
$$

where $d_{M}^{2}(n)$ is the windowing function of size $M, x^{i}(n)$ is the i-th segment of $x(n)$ [2]. The average of $\hat{S}(\omega)^{i}\left(\bar{S}_{x}(\omega)\right)$, for every segment $L$, is the PSD of $x(n)$ according to Equation (3):

$$
\bar{S}_{x}(\omega)=\frac{1}{L} \sum_{i=1}^{L} \hat{S}(\omega)^{i}
$$

The next section will approach the methodology, used in the detection of acoustic signals of PDs, with regard to their evolution.

\section{Experimental Set-Up}

The objective of this research is to evaluate the performance of piezoelectric sensors in the identification of PD evolution. For this, a piezoelectric sensor was attached to the housing of a power transformer, according to Figure $2 \mathrm{a}$ and the following procedure was performed according to the schematic of Figure 2b. 


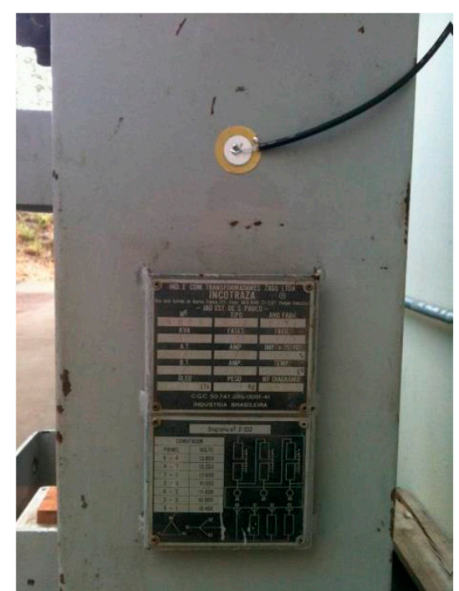

(a)

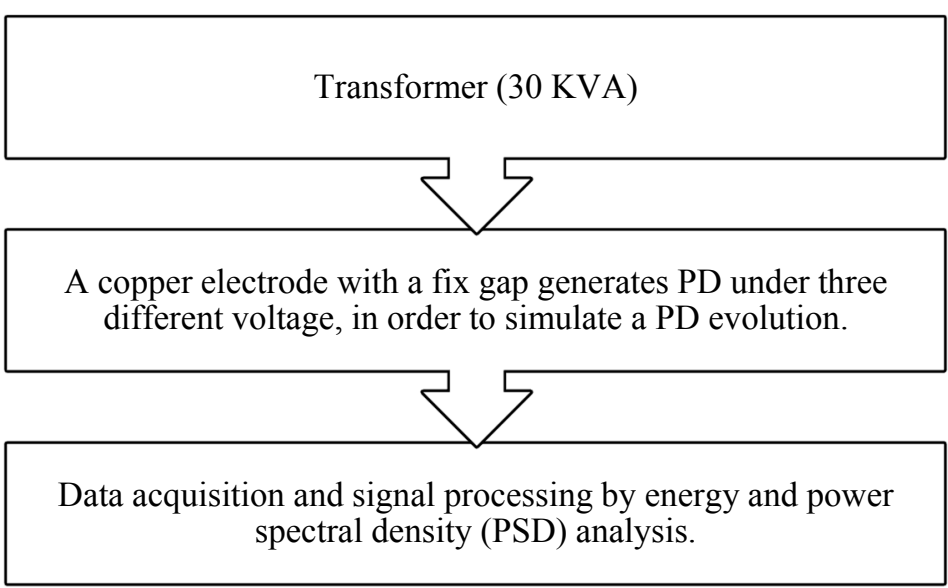

(b)

Figure 2. (a) Sensor coupled to the transformer; (b) schematic diagram of the experimental procedure.

Once the material degradation level and the electric field strength may interfere in the PD incidence, a $2 \mathrm{~mm}$ gap copper electrode was supplied by a $2.1 \mathrm{kV}, 2.8 \mathrm{kV}$, and $3.5 \mathrm{kV}$ voltage source, with the purpose of partial discharge generation. The medium voltage source was provided by a variable transformer set at $30 \mathrm{~V}, 40 \mathrm{~V}$, and $50 \mathrm{~V}$, connected to a transformer with a turns ratio of 70:1. The piezoelectric sensor was attached to the housing of the distribution transformer, through an oleaginous paraffin liquid and the transformer shell was grounded.

For the acquisition of the acoustic signals emitted by the PD, an oscillograph was adjusted to 2 MS/s sampling rate. The cables with grounded shield were used to avoid electromagnetic interference. The sensor signal was amplified 25 times by the INA 128P integrated circuit from Texas Instruments. This integrated circuit has a frequency response of up to $500 \mathrm{kHz}$ band and was used as an anti-aliasing filter. In addition, to mitigate the external vibration effects on the MFC sensor, a high pass digital filter was applied with a cutoff frequency of $20 \mathrm{kHz}$. With these collected data, the energy and power spectral density analysis were performed for each partial discharge level. Ten trials were performed and, since the results that were obtained were similar, only the results of one PD for each level are presented.

\section{Results and Discussion}

Figure 3 shows the PD 1 signal in the time domain, where the voltage applied for the occurrence of the phenomenon, was $2.1 \mathrm{kV}$.

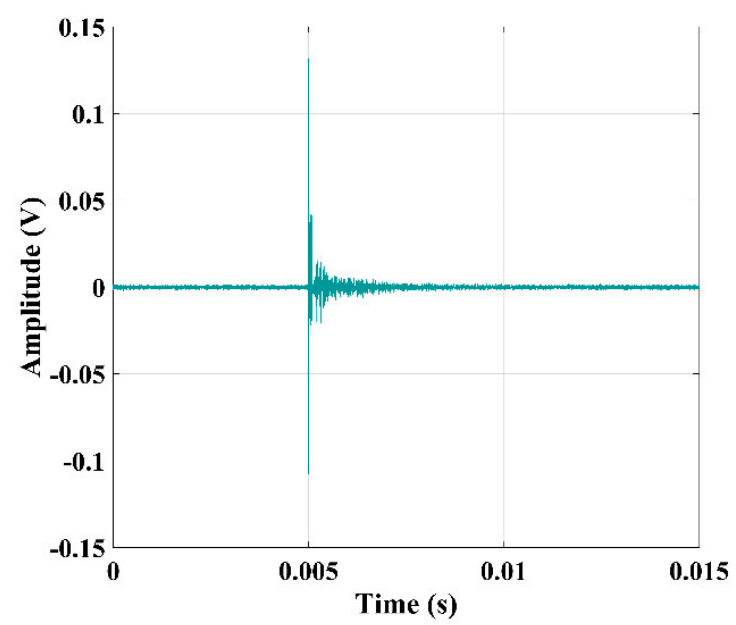

Figure 3. Partial discharge signal in the time domain that was obtained from the piezoelectric diaphragm. 
In Figure 3, the signal provided by the low cost sensor presented an acoustic transient at the time of $0.005 \mathrm{~s}$, with an approximate length of $0.002 \mathrm{~s}$, clearly indicating the occurrence of a PD. Nevertheless, as the objective of this article is to verify the viability of the piezoelectric diaphragm in the identification of the phenomenon evolution, we analyzed the spectral content, as well as the energy of the acoustic signal generated by the three inserted PD. Figure 4 illustrates the power spectral density and the energy for the three signals.

As shown in Figure 4a, the frequency of all acoustic signals that were generated by the three discharge levels is more significant between the range of $20 \mathrm{kHz}$ and $400 \mathrm{kHz}$. In addition, it was observed that the amplitudes of the spectral density increased with the intensity of voltage that was used to generate the phenomenon. For PD 1, the PSD varied around $5 \times 10^{-6} \mathrm{~V}^{2} / \mathrm{Hz}$, increasing to about $1 \times 10^{-5} \mathrm{~V}^{2} / \mathrm{Hz}$, and $2 \times 10^{-5} \mathrm{~V}^{2} / \mathrm{Hz}$, in PD 2, and PD 3, respectively, in the band from $20 \mathrm{kHz}$ to $400 \mathrm{kHz}$. Regarding PD 1, these values represent a growth of two, and four times, for PD 2, and PD 3, respectively. Thus, it can be concluded that, for the same sensor, the power spectral density increases with the intensity of the electric field that was applied to generate each PD.

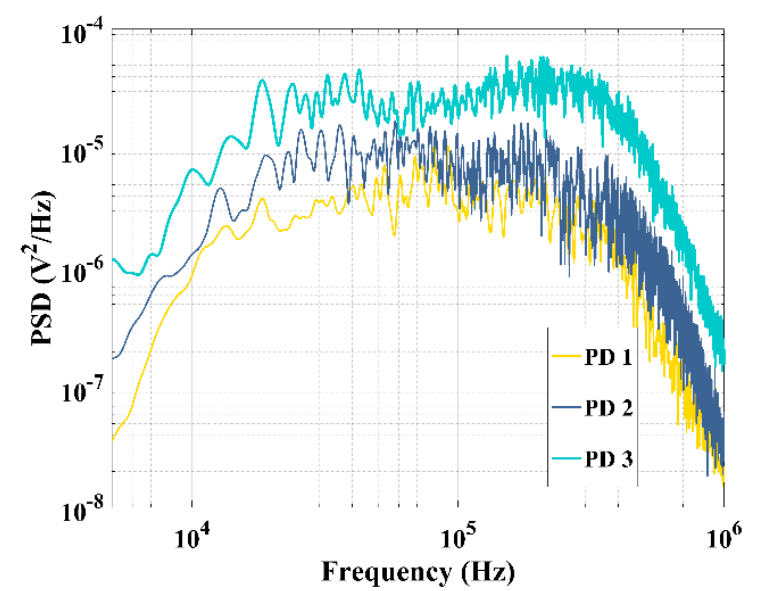

(a)

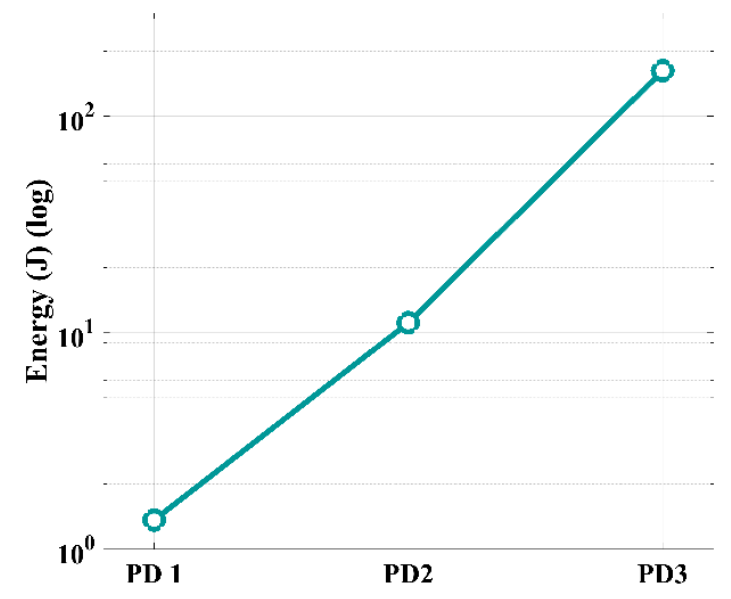

(b)

Figure 4. Power spectral density for different PD levels (a) and energy of acoustic signal for different PD levels (b).

The results presented in Figure $4 \mathrm{~b}$ confirm the results obtained by the spectral analysis. It is observed that energy increases with the intensity of the electric field applied, presenting values of 1.3 J for PD 1, 11.0 J for PD 2, and 160.0 J for PD 3. Regarding discharge 1, the sensor produced signals, with an increase in energy that was 10, and 100 times, for discharges 2, and 3, respectively. In this way, as the intensity of the applied electric field increases, the energy and power spectral density amplitude of the acoustic signal generated by the piezoelectric sensor also increase. Therefore, it can be concluded that the sensor in discussion can be used to verify the evolution of the PD in transformers.

\section{Conclusions}

The employment of piezoelectric sensors in the diagnosis of PD in power transformers, has great importance in guaranteeing a high degree of maintenance performance, as well as the correct operation of the electric system. Thus, this work presented the feasibility study of a low cost piezoelectric transducer in the identification of the level of PD evolution. A $30 \mathrm{kVA}$ distribution transformer was used, and the acoustic signals produced by the PD, were captured by the low cost sensor. For the signal analysis, the energy and power spectral density metrics were used, and based on the obtained results, it was verified that both increased with the intensity level of the applied voltage to produce the discharges under discussion. Therefore, it can be concluded that the low cost sensor can be an effective alternative to measuring the evolution of the PD phenomenon in 
transformers. Therefore, it can be applied in monitoring systems and assist in electrical maintenance planning.

\section{References}

1. Okabe, S.; Ueta, G.; Wada, H.; Okubo, H. Partial discharge-induced degradation characteristics of insulating structure constituting oil-immersed power transformers. IEEE Trans. Dielectr. Electr. Insul. 2010, 17, 1649-1656.

2. Markalous, S.; Tenbohlen, S.; Feser, K. Detection and Location of Partial Discharges in Power Transformers using Acoustic and Electromagnetic Signals. IEEE Trans. Dielectr. Electer. Insul. 2008, 15, 1576-1583.

3. Castro, B.A.; Brunini, D.M.; Baptista, F.G.; Andreoli, A.L.; Ulson, J.A.C. Assessment of Macro Fiber Composite Sensors for Measurement of Acoustic Partial Discharge Signals in Power Transformers. IEEE Sens. J. 2017, 17, 6090-6099, doi:10.1109/JSEN.2017.2735858.

4. Castro, B.A.; Clerice, G.; Ramos, C.; Andreoli, A.L.; Baptista, F.G.; Campos, F.S.; Ulson, J.A.C. Partial Discharge Monitoring in Power Transformers Using Low-Cost Piezoelectric Sensors. Sensors 2016, 16, 1266, doi:10.3390/s16081266.

5. Assagra, Y.A.O.; Gutnik, Y.; Altafim, R.A.P.; Cavalcanti, A.C.; Altafim, R.A.C. Different piezoelectric structures applied to acoustic detection of impulse and corona partial discharges. In Proceedings of the Annual Report Conference on Electrical Insulation and Dielectric Phenomena, Shenzhen, China, 20-23 October 2013; pp. 1242-1245.

6. IEC-TS-62478:2016. High-Voltage Test Techniques-Measurement of Partial Discharges by Electromagnetic and Acoustic Methods; Standard, International Electrotechnical Comission: Geneva, Switzerland, 2016.

7. International Electrotechnical Commission (IEC). IEC 60270-High Voltage Test Techniques, 3rd ed.; IEC: Geneva, Switzerland, 2000.

8. Wang, X.; Li, B.; Roman, T.H.; Russo, O.L.; Chin, K.; Famer, R.K. Acousto-optical PD Detection for Transformers. IEEE Trans. Power Deliv. 2006, 21, 1068-1073, doi:10.1109/TPWRD.2005.861242.

9. Mohammadi, E.; Niroomand, M.; Rezaeian, M.; Amini, Z. Partial discharge localization and classification using acoustic emission analysis in power transformer. In Proceedings of the 31st International Telecommunications Energy Conference (INTELEC 2009), Incheon, Korea, 18-22 October 2009.

10. Álvarez, F.; Garnacho, F.; Ortego, J.; Urán, M.A.S. Application of HFCT and UHF sensors in on-line partial discharge measurements for insulation diagnosis of high voltage equipment. Sensors 2015, 15, 7360-7387, doi:10.3390/s150407360.

11. Ardila-Rey, J.A.; Barrueto, A.; Zerene, A.; Castro, B.A.; Ulson, J.A.C.; Mas'ud, A.A.; Valdivia, P. Behavior of an Inductive Loop Sensor in the Measurement of Partial Discharge Pulses with Variations in Its Separation from the Primary Conductor. Sensors 2018, 18, 2324, doi:10.3390/s18072324.

12. Wang, L.; Cavallini, A.; Montanari, G.; Testa, L. Evolution of PD patterns in polyethylene insulation cavities under ac voltage. IEEE Trans. Dielectr. Electr. Insul. 2012, 19, 533-542.

13. IEEE Standard on Piezoelectricity: An American National Standard; IEEE-ANSI: New York, NY, USA, 1987.

14. Castro, B.A.; Clerice, G.A.M.; Andreoli, A.L.; Campos, F.S.; Ulson, J.A.C. A low cost system for acoustic monitoring of partial discharge in power transformer by Piezoelectric Sensor. IEEE Lat. Am. Trans. 2016, 14, 3225-3231, doi:10.1109/TLA.2016.7587624.

15. Ardila-Rey, J.A.; Montaña, J.; Castro, B.A.; Schurch, R.; Ulson, J.A.C.; Muhammad-Sukki, F.; Bani, N.A. A comparison of inductive sensors in the characterization of partial discharges and electrical noise using the chromatic technique. Sensors 2018, 18, 1021, doi:10.3390/s18041021.

(c) 2018 by the authors. Licensee MDPI, Basel, Switzerland. This article is an open access article distributed under the terms and conditions of the Creative Commons Attribution (CC BY) license (http://creativecommons.org/licenses/by/4.0/). 